amplitude, the attenuation constant being deduced from the readings of the attenuators used to achieve this control. The ratio of measured to theoretical attenuation for plain copper guide and copper or aluminium helix guide was of the order of 1.4 at $35 \mathrm{Gc}$./s. for a 2 -in. diameter guide in all cases,

R. F. Skedd of the Standard Telephone Laboratories discussed the generation, and measurement of attenuation, of unwanted modes associated with long-haul wave-guides, while a paper by Dr. A. E. Karbowiak, G. Craven and V. H. Knight of the same establishment described an equipment for the automatic recording of wave-guide attenuation. This apparatus was capable of recording the variation of attentuation with frequency over the whole of the $40-80 \mathrm{Gc} . / \mathrm{s}$. band in a space of about $\frac{1}{2} \mathrm{hr}$.- an operation which, without the degree of automation used, would require some thousands of independent sets of measurements. An additional paper by Dr. Karbowiak and Mr. Skedd was concerned with an instrument for the accurate determination of relative surface losses in the millimetric band.

Among the other papers, Dr. J. Brown and Dr. A. P. Anderson of University College, London, dealt with the use of a single crystal as a harmonic generator and as a detector at a wave-length of $4 \mathrm{~mm}$. Such an arrangement with the addition of a simple ferrite modulator has been used to measure the impedance of wave-guide components at $4 \mathrm{~mm}$., as well as to determine the permittivity of liquid dielectrics.

W. L. Birch of the General Electric Co. Hirst Research Centre described a milliwattmeter capable of detecting changes of power $\sim 10 \mu \mathrm{W}$. for the 70-80 Gc./s. band in which the power to be measured is absorbed in a carbon losd, the temperature rise in which is detected by a thermopile with which it is in thermal contact. A further wattmeter for use at millimetric wave-lengths was demonstrated by Dr. H. A. French of A.S.W.E., Portsdown, and Prof. A. L. Cullen. The instrument was of the oscillating torque vane type, was capable of measuring power as low as $100 \mu \mathrm{W}$., and, like its predecessors, was also capable of absolute calibration.

The construction of Fabry-Pérot interferometers and their use for the measurement of permittivity of such substances as $\mathrm{TiO}_{2}$ was then considered in two papers by Dr. W. Culshaw and M. V. Anderson of General Telephone and Electronics Laboratories Inc., California, and by Dr. J. S. Seeley and J. C. Williams of Queen Mary College (University of London).

The final session was concerned with measurements on materials. Prof. Barlow described a method for the measurement of permittivity of high-loss dielectrics in which the specimen under test was located in an evanescent guide coupled to a resonant cavity, thus avoiding the disturbance of the field which normally results when the specimen is located within the cavity. For dielectries with small losses Prof. G. B. Walker and S. P. Luthra of the University of British Columbia used 'ghost modes' for the determination of complex permittivity. A disk specimen of the dielectric is located in a wave-guide and a resonant mode of the specimen-the 'ghost' modeis excited, the latter being a mode in which the fields outside the specimen are strongly evanescent and thus contain little stored energy. The limitations of the technique were discussed and a further paper by Prof. Walker and Messrs. J. M. Free and M. E. B. Moffat of the University of Oxford showed how these as well as normal cavity techniques could be used for the investigation of the properties of titanium dioxide and magnesium titanate ceramics at $S, X, J$ and $Q$ bands. Dr. Hamer and $\mathrm{Mr}$. Westcott described a method of measuring the complex permittivity of dielectric films while $R$. B. Nichols and A. E. Brown of Elliott Bros. spoke about an apparatus for grading dielectric cubes according to their exact permittivity. The cubes, used for the fabrication of Luneberg lenses, possess permittivities lying between 1 and 2 which are determined by noting the position at which resonance occurs, as a movable plunger sweeps the cube through a test cavity. Further papers by Dr. Clarricoats and by C. P. Aron of Morganite Research and Development, Ltd., described a simple wave-guide method for the determination of the permittivity of cylindrical dielectric rods and an apparatus for the measurement of the tensor susceptibility of ferrite materials.

G. D. Srms

\title{
PROGRESS IN MICROCHEMICAL METHODS AND TECHNIQUES
}

$\mathrm{T}$ HE 1961 International Symposium on Microchemical Techniques, organized by the Metropolitan Microchemical Society of the United States under the sponsorship of the Commission on Microchemical Techniques, Section of Analytical Chemistry, International Union of Pure and Applied Chemistry, was held during August 13-18, at Pennsylvania State University, University Park, Pennsylvania. The excellent accommodation and facilities made possible an informal atmosphere of fellowship and individual contacts. The purpose of the symposium was to provide a forum for the interchange of information and ideas among technologists from all parts of the world concerning new methods and techniques or unique applications of microchemical or microanalytical interest.

The symposium was inter-disciplinary both with respect to the interests of the almost five hundred technologists attending and the technical programme of ninety-five papers and lectures. The symposium was truly international in character with more than twenty countries represented. Travel of many speakers to the United States was supported by a generous research grant from the National Institutes of Health, U.S. Public Health Service.

The technical sessions and plenary lectures were supplemented by a three-day commercial exhibition of equipment, apparatus, specialities and literature, by six panel discussions, film sessions on the history of microchemistry and research advances, a practical demonstration session, a popular lecture, and by various social events.

The plenary lectures were of salient interest. $\mathrm{H}$ Lieb (Austria) delineated the history of microchemical techniques and A. A. Benedetti-Pichler (United States) the development of microchemistry in the United States. D. Van Slyke (United States) surveyed the evolution of micro-gasometric methods 
of analysis, a field in which he has long pioneered. E. Sawicki (United States) considered the present status of micro methods for the sensitive and selective detection and determination of aldehydes and aldehyde-yielding substances, emphasizing his own successful studies. R. Belcher (England) examined methods developed for the determination of elements and functional groups in organic compounds using sample weights of $30-50 \mu \mathrm{gm}$., an area in which he and his co-workers have made notable advances. N. D. Cheronis (United States) discussed the challenges encountered in the characterization of organic compounds at the microgram and especially the submicrogram range. It appears that many characterization reactions are so slow at concentrations less than about $10^{-5}$ molar that operationally they may be considered as not occurring. The technical papers covered the entire gamut of microchemical and microanalytical interests, and, in elmost all cases, presented novel and previously unpublished research findings.

A special session on thermo-microscopic methods due to L. Kofler was prefaced by a short talk by A. Kofler (Austria), which reviewed the course of her husband's hot-stage experiments. A. Lacourt and N. Delande (Belgium) reported an extended investigation of the identification of some sixteen amino-acids by the hot-stage observation of eutectic temperatures with twenty-five different components, of which oxalic acid and dicyandiamide appear especially good. Of the general papers on microscopy, that of L. W. Gamble (United States) was especially noteworthy; it introduced a new approach for the characterization of aliphatic hydrocarbons, namely, microscopic study of their crystalline urea and thiourea adducts. E. Wiesenberger (Germany) described how the electron microscope could be used somewhat analogously to the light microscope for the detection of substances via the observation of chemical reactions. The reactions, largely precipitation of metal ions with suitable reagents or electrical or chemical reduction to metals, are conducted on the special carriers used in electron microscopy within a field of $0.004 \mathrm{~mm}^{2}$. E. Waldmann (Switzerland) considered the systematic differentiation and identification of crystalline deposits in body tissue by microscopic tests.

Inorganic microanalysis was represented by many significant papers. Those of $H$. Flaschka and P. O. Sawyer (United States) and of A. Ringbom (Finland) emphasized the value of photometric titrations for metal ions present in small amounts or at extremely low concentrations using either ethylenediaminetetraacetate or a chelating chromogenic agent as titrant. Sawyer and Flasehka also described. an elegant micro- and ultramicro-phototitrator using as the light-sensing element a commercial semiconducting photodiode responding in such a small angular range that titrations may be performed in open daylight. J. Zýka (Czechoslovakia) recommended lead tetraacetate as an oxidizing titrant for the potentiometric titration of various metals and organic compounds in acetic acid or in an aqueous medium containing mineral acid. R. Přibil and F. Vydra (Czechoslovakia) based a simple oxidimetric microtitration of cobalt on the finding that iron(III) oxidizes chromium(II) in the presence of 1,10-phenanthroline or $2,2^{\prime}$-bipyridyl, which also masks many interfering cations by complexation. L. Erdey and G. Svebla (Hungary) described the chronometric determination of metal ions based on their catalysis of suitable reactions in which the elapsed time is recorded between mixing and the appearance of the Landolt effect; the peroxide-iodide reaction permits the determination of $0.1 \mu \mathrm{gm}$. of molybdenum within 99 per cent accuracy.

Quantitative elemental organic analysis, as a major subject for microchemical research, was considered in many papers. In the combustion analysis for carbon, hydrogen and sulphur, C. Maresh and co-workers (United States) proposed a gas chromatographic finish. H. Malissa and E. Pell (Austria) reported on their recent investigations on the complete automation of the micro-determination of carbon, hydrogen and sulphur. A. M. G. Macdonald and Y. Gawargious (England) gave the results of a comparative study of various high catalytic fillings in the combustion analysis for carbon and hydrogen; cobaltic oxide appeared to be the most satisfactory for general work. J. A. Kuck and co-workers (United States) described an instrumented combustion method for carbon and hydrogen in which carbon dioxide is assessed by infra-red absorptiometry and hydrogen by thermal conductivity.

G. Ingram (England) introduced a direct ignition in an oxygen-filled chamber at $850^{\circ} \mathrm{C}$. for the determination of carbon, hydrogen, halogens and sulphur; the combustion products are swept into appropriate absorbing reagents and determined. The method requires only $30 \mathrm{~min}$. or less per sample and is applicable to difficult materials, such as organic fluorine or phosphorus compounds. W. G. Guldner and A. L. Beach (United States) based a rapid micro determination of oxygen on pyrolysis of the organic sample in a helium stream in an induction-heated graphite crucible and oxidation of the carbon monoxide, after trapping of unsaturated compounds, to carbon dioxide, which is absorbed in barium hydroxide solution and determined conductometrically. J. L. Brownlee, jun. (United States), presented an intriguing approach to the determination of oxygen in organic compounds involving a neutron-induced, reaction in an atomic reactor, namely: ${ }^{6} \mathrm{Li}(n, \alpha)^{3} \mathrm{H}$; ${ }^{16} \mathrm{O}(t, n)^{18} \mathrm{~F}$; fluorine-18 is separated and determined radiochemically.

Noteworthy prpers in organic functional group analysis included that of J. E. Zarembo and M. M. Watts (United States) on the determination of the carbodiimide function, of $\mathrm{E}$. Sawicki and co-workers (United States) on a highly sensitive spectrophotometric determination of azuelene, and of W. Merz (Germany) on the determination of active hydrogen using alkyl-substituted metal aluminium hydrides. C. A. Rush and J. I. Miller (United States) proposed barium isopropyl methylphosphonate as a rigorous reference standard for carbon, hydrogen, phosphorus, barium and alkoxyl. S. R. Palit and P. Ghosh (India) based a determination of acids, bases and salts at micromolar concentrations in benzene on the colour behaviour with rhodamine and certain other dyes previously extracted by benzene under specific conditions. M. Bobtelsky (Israel) considered his recent research work with microheterometry in work on the reactions and nature of large organic molecules, including dye lakes and metal-protein complexes.

Physical measurements on the micro scale also received attention. The use of a dynamic flow technique in place of the usual static drop method was recommended by C. L. Ogg and co-workers (United States) for the thermistorized determination of molecular weight. W. Simon and co-workers 
(Switzerland) described refined thermistor cireuitry and instrumentation for the routine, precise determination of molecular weight using a sample weight of only $2.5 \mathrm{mgm}$. for a molecular weight of 500 . P. D. Garn and J. E. Kessler (United States) demonstrated that thermal analysis is feasible for sample weights less than $1 \mathrm{mgm}$. by the use of appropriately designed sample holders.

In the session on nucleonics, G. F. Morrison and J. F. Cosgrove (United States) described the application of neutron activation to the sensitive determination of the copper activator in single crystals of electroluminescent zinc sulphide weighing less than $500 \mu \mathrm{gm}$. B. A. Thompson (United States) considered how radiochemical techniques and especially neutron activation could be applied to the determination of the principal components, trace impurities and thickness of metal films a few angstroms in thickness and one square centimetre in area; such films are encountered in the performance and design of electronic devices, including vacuum tubes. M. T. Kelley (United States) reviewed the present status of the analysis of highly radioactive reactor fuels and spent products using liquid samples of 5-700 $\mu \mathrm{l}$.; coulometry, spectrophotometry, flame photometry, emission spectroscopy, titrimetry and radiometry, and density measurements are feasible by remote controlled, automatic or 'slave' manipulated operations. A. Holasek and co-workers (Austria) explained the difficulties and operational problems encountered in the synthesis of milligram amounts of fatty acids and esters labelled with carbon-14, and considered salient factors involved in the selection of suitable synthetic methods.

Chromatographic methods were the subject of many interesting papers. E. G. Wollish (United States) considered critically the accelerating interest in alumina and silicic acid for thin-layer chromatography in the separation and identification of organic compounds, including drugs. H. K. Mangold and co-workers (United States) exemplified how thinlayer chromatography acts as a valuable analytical and preparative tool in lipid chemistry. L. S. Ettre and W. Averill (United States) demonstrated how new gas chromatographic instrumentation using capillary (Golay) columns and ultra-sensitive detectors permit the direct analysis of trace components in samples of micro-size, and set forth criteria for the selection of methods and instrumentation. L. Wiesner and W.-J. Schmidt-Küster (Germany) described the gas-chromatographic performance of a new integrating counter-detector using a flow-type proportional counter containing a source of ionizing radiation and used with a suitable scaler. T. S. Ma and M. Gutterson (United States) recommended gas chromatography in the determination of several organic functional groups. S. Patton (United States) considered the identification of food flavours using a diversity of techniques including gas, paper and column chromatography, and the study of the dinitrophenylhydrazones of volatile keto-compounds. Paper chromatography was applied by B. Bobrański and L. Syper (Poland) to the identification of drugs containing unsaturated radicals following reaction with mercuric acetate. A. K. Dey and E. J. Singh (India) explained the behaviour and application of various complexing agents in the separation of metal ions by the Weisz ring-oven technique.

The contemporary position of small-scale methods in the teaching of chemistry at the secondary school and collegiate levels was assessed in a special session with speakers from Northern Ireland (C. L. Wilson, N. N. Gilpin), England (J. B. Headridge, H. Holness), and the United States (N. D. Cheronis, T. S. Ma and J. A. Kuck). A. Steyermark (United States) considered the training of chemists in industry in quantitative organic analysis.

When the Proceedings of this symposium become available as a single volume (Interscience, early 1962), the interested technologist will be able to ascertain the position of microchemistry and microanalysis for 1961 in the spectrum of scientific activity, and to comprehend the research trends that will yield the 'to-morrow' of these disciplines. In brief, microchemical and microanalytical research faces continuing challenges with ever smaller samples, ever more minute components, coupled with demands for adequate accuracy, precision and rapidity. Improved instrumentation, automation and new techniques and reagents, coupled with re-assessment of present methods and approaches, will energize further advances.

A. J. BARNARD, JUN. JoHN Y. STEEL

\section{INSECT POLYMORPHISM}

\begin{abstract}
A SYMPOSIUM on polymorphism in insects, organized by the Royal Entomological Society of London, was held at the Imperial College of Science and Technology, London, during September 21-22. The president, Prof. G. C. Varloy, was in the chair at the first meeting and expressed a hope that special attention would be directed to the agencies promoting and controlling this type of variation.

Prof. O. W. Richards, in a general introduction, stated that polymorphism is very widespread among the insects and occurs in all the major orders. The metamorphosis of insects, itself a mode of polymorphism, perhaps makes them especially prone to such variation. There are two principal types of polymorphism: the genetic type, where two or more genotypes co-exist in a state of balance, and the induced type, in which some variable environmental factor such as population-density, food, day-length
\end{abstract}

or temperature leads to the development of two or more kinds of individual in a species.

Dr. E. B. Ford, in an account of the theory of genetic polymorphism, stated that the standard examples are only special cases of a more general phenomenon which is often overlooked when the characters involved are not easily visible. This is the tendency for selection to favour the heterozygote over both homozygotes when, after some change in the environment, a rare major gene begins to spread. The favourable effects of the gene will tend to become dominant and the unfavourable ones recessive, and the heterozygote will have all the advantages and none of the disadvantages of the homozygotes. Where polymorphism results from the interaction of several genes, the whole complex is controlled by a supergene, the link between the units of which may be strengthened by a chromosome inversion. The original effects 